\title{
La constitución del sujeto en la Odisea de Homero. Odiseo profano: la aletheia, el lenguaje y el êthos ${ }^{1}$
}

The constitution of the subject in the Odyssey. Unholy Odisseus: the Aletheia, the language and Ethos

Si pudiera apartar la magia de mi sendero, olvidar enteramente las formas mágicas, estar ante ti, Naturaleza, un hombre solo, entonces sí valdría la pena ser un hombre.

(Goethe)

\section{Resúmen}

El presente artículo pretende realizar un análisis de la constitución del sujeto en el poema épico la Odisea de Homero. El argumento central explora la posibilidad de la constitución del sujeto humano a partir del espacio abierto, no codificado y no reglado en el que se desarrolla la obra, y que permite la articulación de tres momentos específicos, tres encuentros que llevan al héroe a poner en escena el coraje de su decisión ética, con tres elementos necesarios para la constitución de la subjetividad, a saber, la aletheia (la verdad), el lenguaje y el êthos.

Palabras Clave: sujeto, Odisea, ética, moral, aletheia, lenguaje.

\section{Abstract}

This paper attempts an analysis of the constitution of the subject in the epic poem the Odyssey. The central argument explores the possibility of the constitution of the human subject from the not regulated, uncoded open space in which the poem takes place, which allows the articulation of three time points, three meetings to lead the hero to put in scene the courage of their ethical decision, with three elements necessary for the constitution of subjectivity, namely the aletheia (truth), language and êthos.

Keywords: subject, Odyssey, ethic, moral, aletheia, language.

\footnotetext{
1 El presente artículo es un producto del proyecto de investigación titulado "La violencia de estado en el conflicto colombiano. Una revisión desde las categorías políticas de Giorgio Agamben y Hannah Arendt", realizado en el grupo de investigación Lumen Humanitas y financiado por la dirección de investigaciones de la Fundación Universitaria Católica Lumen Gentium (UnICATólicA).

2 Docente investigador de tiempo completo de la Fundación Universitaria Católica Lumen Gentium. Docente de la Universidad del Valle, Cali, Colombia. Psicólogo de la Universidad del Valle y magister en Filosofía de la Universidad del Valle, Cali, Colombia. Correo electrónico: fjrojas@unicatolica.edu.co
} 
En el presente capítulo no se pretende hacer un análisis detallado y erudito sobre un tema que, por lo demás, a nivel del desarrollo histórico ha tenido un tratamiento teórico abundante desde diferentes perspectivas: antropología, lingüística, derecho, filosofía, literatura, entre otras. El propósito es realizar un análisis de la constitución de la subjetividad y el êthos, en una relación interdependiente, a la luz de tres fragmentos específicos - "tres encuentros"- de la poesía heroica en Odisea de Homero que permiten identificar tres elementos cardinales de esa constitución. La idea consiste en realizar una lectura de la Odisea como condición de posibilidad del nacimiento del sujeto humano a partir de una constitución particular del êthos en el ámbito de lo no previsto, no codificado y no reglado. Dicho en otros términos, demostrar la capacidad del ser humano para ponerse fuera de sí, representarse en otro, salir de su mundo (en la Ilíada un mundo sistematizado y conocido, construido mediante reglas fundamentales que lo ordenan con un límite incluso espacial) para entrar en otro mundo; un mundo desconocido que surge como exigencia ética para una constitución determinada de la subjetividad.

Mucho se ha dicho sobre las ineludibles diferencias entre las dos obras asignadas al mismo poeta, la Ilíada y la Odisea, estas han llevado a pensar que quizás su autoría se debe asignar a diferentes sujetos o creadores; el autor no sería el mismo. Pero entre las diferencias que se pueden establecer es posible encontrar un material de riqueza sutil que permite cavilar a fondo sobre las relaciones entre la constitución subjetiva y el pensamiento. Más importante que "devanarse los sesos" intentando descubrir la identidad del autor, el aspecto esencial es el contenido de la obra. Finley (1961) sugiere, entre otras diferencias, que la más radical consiste en las relaciones que se instituyen entre los héroes y los dioses. Los dioses intervienen solo de manera esporádica en la Ilíada mientras que en la Odisea su presencia actuante se encuentra desde el inicio y permanece constante durante todos los cantos:
[...] comienza en los cielos con la invocación de Atenea a Zeus para que dé fin a las pruebas del héroe, y termina cuando la diosa hace cesar la sangrienta contienda entre el héroe y los parientes de los pretendientes a quienes había dado muerte. (Finley, 1961, p. 35)

Es esta diferencia la que permite hacer un análisis que se distancia de la observación infinitamente válida de Finley cuando refiere que en la Odisea se sustituyó el elemento personal -caracterizado por el agrado o desagrado de los dioses hacia los héroes en la Ilíada- por las exigencias de la justicia. Se propone entonces que esas exigencias surgen sobre la base de una exigencia ética y se intenta demostrar el porqué.

\section{Ética y moral en la Odisea}

En una primera aproximación, y siempre teniendo como norte la relación entre la constitución subjetiva y el êthos, se podría volver sobre la ya releída y rediscutida diferencia entre ética y moral, esta vez para pensarla como una genealogía en el poema épico de Homero. La moral se instaura en el sujeto como un código, dicta, legisla, establece pautas de comportamiento, y le impone al individuo un modelo de conducta que representa las obligaciones y deberes que debe asumir en el trayecto de su vida. Por tanto, se puede afirmar que la moral no busca lo establecido, sino más bien, establecer, fijar, codificar; busca un ordo, un orden que recordará, entre otras cosas, a las formaciones de los guerreros aqueos durante las batallas.

La moral vista así no dejaría posibilidad para la deliberación. La ética por el contrario, no se basa en un código -escrito o no- que reglamente la conducta humana, no se remite a un mandamiento o a un ser superior que dicte lo que se debe hacer; la ética implica una relación del sujeto consigo mismo, el sujeto se hace cargo de sí y "se responsabiliza de sí mismo, en total independencia de toda autoridad, costumbre 
o presión social. Por eso, la ética es el terreno por excelencia de la decisión, de la elección" (Sampson, 1998) ${ }^{3}$.

Ya Aranguren (1998) había declarado en un famoso ensayo sobre la etimología de la ética, que el análisis filológico de esta palabra conlleva la reflexión de dos fuentes desde las que se puede evaluar tanto su sentido originario como su confusión histórica, estas dos fuentes son la griega y la latina. Aranguren aclara con Heidegger que muchos de los conceptos filosóficos griegos más importantes oscurecieron su sentido originario en su traspaso al latín. Probablemente la confusión actual que se reproduce en los diversos discursos científicos, filosóficos, jurídicos, etc., entre la palabra moral y la palabra ética, sea un producto de este avatar importante en la historia occidental que resulta del encuentro entre Grecia y Roma ${ }^{4}$.

En su concepción griega original, êthos se entendía como morada, hábitat. Ya desde la Carta sobre el bumanismo de Heidegger (2000/2006) pasó a tener una vinculación directa con la ontología, por esta razón êthos tiene el sentido de la manera en la que el humano se habita a sí mismo, forma relaciones consigo mismo y genera una forma de relación particular con el mundo. Junto con Heidegger sería posible decir que el êthos es la morada del ser, sin embargo, en griego una palabra comúnmente relacionada con êthos es éthos, más relacionada con el concepto griego de héxis que implicaba de

3 El análisis que se realiza en esta parte de la investigación sobre la moral y la ética estriba sobre el escrito del profesor Sampson, el cual se considera esencial para poder adentrarse en la diferencia entre los dos términos.

4 Una de las confusiones más comunes, en la que suelen caer muchos profesionales y académicos, es la de homologar ética y deontología, es común escuchar a los profesionales de diversas disciplinas hablar de los "códigos de ética". Advertimos aquí el peligroso y nada fructífero oxímoron según los argumentos antes expuestos. Los códigos normativos de disciplinas como la medicina o la psicología, entre otras, son códigos deontológicos, es decir que se fundamentan en el "deber ser", dicho en otros términos, la deontología busca normalizar pautas de comportamiento según el deber. La deontología es un código moral. Por el contrario, si la ética es el lugar por excelencia de la elección sobre la propia manera de vivir, ésta no puede estar constreñida a un código, a una normalidad estadística, ni mucho menos a un "deber ser". La ética es entendida aquí en su sentido originario como "forma-de-vida". La ética escapa al ámbito de las consideraciones jurídicas que es el terreno propio de todo código moral. La forma-de-vida tiene siempre en potencia la libertad de no someterse a ningún código. manera aproximada, el hábito que se adquiere por repetición o costumbre.

En el paso del concepto griego de êthos al latín, los romanos -que en palabras de Aranguren tenían poca actitud para la filosofía- tradujeron los dos conceptos (êthos y éthos) con un único término del reservorio de su lengua, a saber, mors del que procede nuestro concepto de moral. El êthos vertido al latín mors perdió su sentido como forma de vivir para pasar a gravitar ante la idea de un carácter adquirido por hábito, de allí la importancia de las costumbres como insumo de la acción correcta, y posteriormente, de una concepción de las acciones en su polaridad de "buenas" o "malas" acciones.

En el análisis del presente artículo, se toma al êthos en su estricta concepción semántica como forma de vida y no como un término ligado a la acción moral que no solo parte de las costumbres sino del código que pretende regular y normalizar la conducta de los hombres. Esta concepción permite -entre otras- una relación más específica con la idea de una construcción del sujeto, ya que este se entiende como el producto de las relaciones del individuo (especie biológica) con su cultura (condición de existencia simbólica) y evita cualquier reducción, ya sea esta una reducción biológica o una reducción cultural. El sujeto no es el individuo, pero tampoco es una mera construcción cultural. Si se quiere, el sujeto es el producto irreductible de la historia de encuentros y desencuentros entre la naturaleza y la cultura y por esta misma razón, es un elemento que escapa a todo intento de normalización a partir de leyes naturales o leyes culturales, su núcleo ético como forma de vida es la consideración singular del sujeto5.

Realizar esta aclaración cobra relevancia porque lo que se encuentra como contraste principal entre los dos poemas épicos es algo del orden

5 Para una revisión más extensa en la vía de concebir la ética como una forma de vida se recomiendan los siguientes textos: Maclntyre (1991), Hadot (1998) -por sus relaciones entre la ética como forma de vida y el sujetoFoucault (1999; 2002; 2009; 201 1), Lacan (2009), Rajchman (1991), Rojas (2014; 2015) 
de la diferencia: un mundo codificado, un mundo reglado; en la Ilíada hay un mundo en el cual la inminencia de la batalla hace parte de su fundamento, de su propia existencia como obra. La guerra, el honor y el carácter heroico estaban codificados, un mundo en el cual los límites espaciales, el gigantesco mar y las murallas de Troya restringían la elección de los héroes.

La Odisea se muestra como otra -obra-, un mundo otro, no codificado de antemano. El destino del héroe -de Odiseo- no está entre el mar y las murallas de Troya como entre Escila y Caribdis, el destino no está escrito, se construye sobre la base de su decisión ética como sujeto. De qué otra forma podría explicarse que en la Odisea el héroe sea uno solo y que su espacio de acción sea marino, "dominio donde reina el azar, la variabilidad de condiciones en un espacio polimorfo y moviente, que expone a los que se aventuran en él a peligros innumerables" (Vega, 2000). Lo que exige este espacio polimorfo es el uso de algo que va más allá de la fuerza y la habilidad en la batalla mostrada por los héroes en la Ilíada, exige una especie de astucia representada por el nombre de la diosa Metis y que también implica -en su polisemia- la capacidad para tomar decisiones en el Kairos en el momento oportuno, la capacidad para desprenderse de sí, para encontrarse o encontrar su "verdad" a través de un tipo específico de curiosidad.

\section{Los tres encuentros}

¿Por qué hablar de polisemia? Precisamente porque la Metis -atribuida en este caso a Odiseose manifiesta de formas polimorfas de acuerdo con lo imprevisible de la situación que se presenta en un momento determinado. Sobre la base de esta afirmación se proponen tres escenas, el dibujo de tres encuentros esenciales en la obra que permiten poner de manifiesto la relación entre el êthos -el lugar de la decisión- y la constitución de Odiseo como sujeto.

\section{Primer encuentro: Odiseo y la flor del loto}

Después de navegar errante durante nueve días, Odiseo vislumbra la tierra de los lotófagos, hombres que solo se alimentaban de la flor del loto, ¿sus efectos? Tal vez no muy alejados de lo que Huxley (2014) representara como el "soma" en su interesante novela Un mundo feliz, por lo menos allí subyace el mismo principio ${ }^{6}$. Decía Homero (2000): "el que de ellos probaba su meloso dulzor, al instante perdía todo gusto de volver y llegar con noticias al suelo paterno; solo ansiaba quedarse entre aquellos lotófagos, dando al olvido el regreso, y saciarse con flores de loto" (Canto IX, pp. 93-97).

Al igual que en la novela de Huxley, alimentarse del loto significa simplemente dejar de ser, olvidar no significa solamente olvidar lo que estaría en la superficie y que en Odiseo pudiera significar olvidar las vicisitudes por las que atraviesa -lo cual sería un alivio para su alma golpeada por lo imprevisto, como las olas golpean a los peñascos-, olvidar significaría borrar de la memoria su historia y el deseo que lo anima; sería, en síntesis, olvidar la verdad (alétheia) que lo sostiene y lo fundamenta como sujeto.

Odiseo se encuentra en un mundo desconocido, no codificado. La decisión de volver a las naves y negarse a olvidar o a ocultarse a sí mismo es un intento por encontrar su mundo y "encontrar su mundo significa - para un pueblo y para un individuo- tanto como encontrarse a sí mismo, alcanzar la realización del ser propio" (Otto, 2003, p. 29). Encontrar su propio ser en el deseo que lo anima, su patria, su esposa, su hijo, el deseo que es su verdad como sujeto, es, después de todo, su decisión ética basada en su alétheia que se erige contra el olvido (lethé) (Allouch,

6 Sería interesante analizar cuáles serían los malestares modernos y también la manera en que los dispositivos de poder actuales crean formas microfísicas para la cura de esos males. En un análisis de este tipo no se pueden dejar de lado los llamados trastornos mentales consignados en los manuales diagnósticos y estadísticos de la psiquiatría moderna, y el tratamiento farmacológico con el que se les pretende curar; la industria farmacéutica actual es el dispositivo encargado de la producción en masa del "soma" contemporáneo. 
1998, p. 148). No se debe olvidar también que esta "verdad", esta alétheia, en una de las cuatro definiciones que da Foucault, se refiere al alethés como lo que "existe y se mantiene más allá de todo cambio, lo que persiste en la identidad, la inmutabilidad y la incorruptibilidad" (Foucault, 2010, p. 233). Es entonces, desde la posición de Foucault, una verdad "recta" que no tiene desvíos, que no tiene mezcla y mantiene su identidad. No es sorprendente entonces, que la decisión de Odiseo al negarse a la lotofagia sea una muestra de mantener incólume su "verdad" y rechazar la posibilidad de mezclar su identidad con el loto.

\section{Segundo encuentro: Odiseo y Polifemo}

Luego de haber dejado la tierra de la lotofagia y la lethé $e^{-7}$ su decisión lo encamina -sin saberlo de antemano- hacia la tierra de lo apolítico, su encuentro se da con otro mundo, pero en esencia con otro, otro que no se configura como un alter, un individuo sin ley ni justicia que no puede funcionar como la imagen especular del héroe. Pero este encuentro se da sobre los límites de la curiosidad, palabra que puede remitir -sin duda alguna- a uno de los significados de la que ya se apuntó en este escrito como polisémica: metis. ¿Es acaso cualquier curiosidad a la que aquí se hace referencia y de forma osada se incluye bajo este epíteto tan importante de metis? De ninguna manera. En la introducción a su obra El uso de los placeres, Foucault se refiere a esta curiosidad como:

[La] única especie de curiosidad, por lo demás, que vale la pena practicar con cierta obstinación: no la que busca asimilar lo que conviene conocer, sino la que permite alejarse de uno mismo. ¿Qué valdría el encarnizamiento del saber si solo hubiera de asegurar la adquisición de conocimientos y no, en cierto modo y hasta donde se puede, el extravío del que conoce? (Rajchman, 2001, p. 155)

La historia de Odiseo es la historia de un extravío, ¿acaso no es esta curiosidad que describe Foucault la que guía al héroe hacia lo imprevisto

7 Lethé puede ser traducido aproximadamente como "olvido", también como lo "oculto". Así, la palabra griega Aletheia, que se ha traducido como "verdad", sería lo no olvidado o lo no oculto. a costa de perderse a sí mismo?, perderse para encontrarse o encontrarse negándose a sí mismo. Esa curiosidad, esa particular "voluntad de verdad", y aquí se ve aparecer nuevamente la alétheia, lo lleva a explorar la isla para conocer quiénes son los "otros" que viven ahí, para des-ocultar. Su encuentro con el cíclope Polifemo es un encuentro con la ausencia de la ley, la ausencia del gran otro que se revela en el vacío que posee en el lenguaje, en contraposición del héroe Odiseo que se descubre y a la vez descubre el poder ambivalente del lenguaje ${ }^{8}$. Luego de haber urdido el plan contra el cíclope sin ley, el héroe adelanta el primer paso:

Preguntaste, cíclope, cu[áll era mi nombre glorioso y a decírtelo voy, tú dame el regalo ofrecido: ese nombre es ninguno (oudeís). Ninguno (oudeís) mi padre y mi madre me llamaron de siempre y también mis amigos. Tal dije y con alma cruel al momento me dio la respuesta: a "ninguno" (oudeís), me lo he de comer el postrero de todos, a los otros primero, hete ahí mi regalo de huésped. (Homero, 2000, canto IX, pp. 364-370)

Es así como en su encuentro con Polifemo, Odiseo llega -a través del uso del lenguaje y de su ley formal- a vencer a aquel que no posee ley alguna, aquel ser apolítico cuyo logos está ausente. Adorno dice: "Odiseo se afirma a sí mismo negándose a sí mismo como nadie (oudeís), salva su vida haciéndose desaparecer" (Adorno, 2007, p. 73). La gran paradoja queda instaurada -tal como se podría interpretar en Foucault-, ese proceso de constitución de su propia subjetividad se da a través de un alejamiento, un distanciamiento de sí que solo se puede hacer por la existencia del lenguaje.

\section{Tercer encuentro: Odiseo y el canto de las sirenas}

Este encuentro está marcado por la decisión que se basa en la misma curiosidad que guía toda la

8 Para revisar en detalle esta doble función del lenguaje que se refleja siempre entre ausencia y presencia, aparecer y desaparecer, fort-da y la estructura fundamental del lenguaje humano como deixis, se deben revisar algunos autores del llamado estructuralismo. Se recomienda particularmente la obra de Benveniste (1971). 
historia del héroe homérico, aquella particular "voluntad de verdad" que lo lleva a elegir en un momento crítico no codificado de antemano, elección que se transforma, no solo en el encuentro de sí mismo, sino también en la muerte del mito. El canto de las sirenas:

\begin{abstract}
Llega acá de dánaos honor, gloriosísimo Ulises, de tu marcha refrena el ardor para oír nuestro canto, porque nadie en su negro bajel pasa aquí sin que atienda a esta voz en dulzores de miel de los labios nos fluye. Quien la escucha, contento se va conociendo mil cosas: los trabajos sabemos que allá por la Tróade y sus campos de los dioses impuso el poder a troyanos y argivos y aun aquello que ocurre doquier en la tierra fecunda. Tal decían exhalando dulcísima voz y en mi pecho yo anhelaba escucharlas. Frunciendo mis cejas mandaba a mis hombres soltar mi atadura; bogaban doblados contra el remo y en pie Permedes y Euríloco, echando sobre mí nuevas cuerdas, forzaban cruelmente sus nudos. (Homero, 2000, canto XII, pp. 185-195)
\end{abstract}

¿A qué se asiste en este fragmento? A la fijeza del mito, a su ritual continuo que asegura su existencia, nadie puede pasar por aquel siniestro lugar, escuchar a las sirenas y salir ileso de su destino fatídico. Nada hay de extraordinario en no escuchar el mito de melodía seductora en la boca tentadora de estos seres porque los oídos están repletos de cera, pero la elección de Ulises implica un acontecimiento -a todas luces- transgresivo, único y de una consecuencia indudable para sí mismo y para la historia. A riesgo de extraviarse, de perderse en el canto o en el fondo del mar, su decisión implica pensar de otro modo, constituirse en lo imprevisible, demostrar que las cosas pueden ser de otra forma: "cada una de las figuras míticas está obligada a hacer siempre lo mismo, cada una existe por su repetición, el fracaso de esta significaría su fin" (Adorno, 2007, p. 71).

Odiseo elige escuchar el mito, desafiarlo y superarlo, el mito se destruye porque su repetición fracasa, se supone que nadie que escuchara la voz de las sirenas podría escapar a su destino. El mito es la perpetuación de lo mismo, es el paradigma de lo idéntico, por el contrario, el héroe es el paradigma de lo otro, de otra verdad y de otra vida, construye su propio destino de la misma manera en la que se construye a sí mismo, en el acontecimiento, y lo hace a través de la polisémica metis. Adorno apunta: "el órgano del sí mismo para superar aventuras, para perderse a fin de encontrarse, es la astucia" (Adorno, 2007, p. 62); dicho en otros términos: la metis.

Parece claro, aunque sea solo un sobrevuelo esquemático, que toda la obra representa, no solo la superficialidad de la intervención de los dioses, la magia y la hechicería -elementos que son un recurso adecuado pero, que no estarían allí de no ser por la función que cumplen como perpetuadores de lo idéntico-, y la lucha agónica constante que establecen con lo "otro". Dicho en otros términos, se transforma el mundo conocido y codificado en extremo, en un mundo desconocido e imprevisible en el cual la elección ética por "naturaleza" pone a prueba la constitución subjetiva. $\mathrm{Y}$ en esa constitución subjetiva, ¿cuáles son los recursos para vencer lo imprevisto?, ¿para negarse a seguir un destino que se repite? En primer lugar, la verdad (alétheia) que se erige contra el olvido (lethé) y contra la mezcla de la incorruptible identidad. ¿El olvido de qué?, del deseo que lo instituye como sujeto. En segundo lugar, el lenguaje que sin querer parecer demasiado determinista, se erige como el punto posible del encuentro de sí a través de una operación doble: de alejamiento y acercamiento, la muerte de la identidad que asegura la vida, el develamiento del sí mismo a través del ocultamiento necesario 9 y por último, no es claro acaso, que la decisión ética constitutiva de la subjetividad se pone en juego a través de la destrucción de lo que se creía indestructible, la posibilidad de pensar que "yo" puedo ser de otra forma, ser y estar en un lugar diferente al de las determinaciones exteriores que se imponen como un mito eterno y repetitivo, y precisamente, esta operación ética radical no se podría dar en el mundo de lo conocido y ordenado de antemano,

9 Acaso no será esto lo que se ve en el psicoanálisis, se habla para ocultar algo, que sin embargo termina por ser descubierto y al hacerlo, se manifiesta allí, desnuda, la subjetividad. 
solo se puede dar en medio de una crisis (krisis), en un acontecimiento no preestablecido y para el que nada nos ha preparado. La última palabra la tiene la representación de Auschwitz que demuestra de manera clara lo que aquí se pretende decir ${ }^{10}$.

Llegados a este punto, no sobra preguntarse cuál es el horizonte ético que le corresponde al sujeto contemporáneo. ¿Acaso no asistimos en nuestros días a la confiscación de todas las actividades del mundo humano por los dispositivos del poder?, ¿acaso no es claro que vivimos en un mundo codificado en extremo y decidido por los más variables dispositivos de subjetivación? ¿Si el êthos es una potencia constitutiva de toda forma de vivir, cuáles son las maneras contemporáneas de convertir esa potencia en acto?

En un interesante texto titulado el Homo Sacer. El poder soberano y la vida nuda, Agamben (2010) aventura una hipótesis en la cual asevera que aquel núcleo jurídico político que hizo posible las masacres de los campos de concentración en la Alemania nazi fue la figura jurídica del "Estado de excepción", una figura del derecho que implica la posibilidad de toda nación de poner en suspensión los derechos que cobijan a sus ciudadanos para responder con nuevas normas a un estado de emergencia; no sobra recordar que los primeros campos que surgieron en Alemania se concibieron como campos de protección que luego desembocaron en una realidad contraria. Se vislumbra un panorama nada prometedor, a través de un análisis profundo Agamben (2010) devela el estado jurídico-político en el que se encuentran nuestras actuales Naciones-Estado: el estado de excepción se ha convertido en regla.

En un mundo en el que el derecho se erige en una lucha agónica con las formas de vida, en el cual el precio a pagar para el retorno de la normalidad jurídica y mental está decidido de una vez y para siempre desde la razón universal -sea

10 Cf. La magnífica obra de Agamben (2000) en la que la constitución ética y subjetiva se da en el marco de la situación extrema e imprevisible de la figura espantosa -monstruo mítico- de los campos de concentración. esta deontológica o psiquiátrica y farmacológica-, el olvido del sujeto debe dar paso al retorno del sujeto en la recuperación perentoria que realiza del "uso" de su propia vida. "Profanar" en la obra de Agamben (2009) significa invertir el estado normativo de las cosas, recuperar los usos de aquello que ha sido confiscado, poder un poder. Se debe considerar -de manera ineludible- que profanar es la tarea política por venir. Si se formula la pregunta sobre la posibilidad de la emergencia de un sujeto ético en los tiempos que corren, este sin duda, vendrá a la manera de un Odiseo profano.

\section{Referencias}

Adorno, T. W. (2007). Dialéctica de la Ilustración, obra completa. Madrid: Akal.

Agamben, G. (2000). Lo que queda de Auschwitz. El archivo y el testigo. Homo Sacer III. Valencia: Pre-Textos.

Agamben, G. (2009). Profanaciones. Buenos Aires: Adriana Hidalgo Editores.

Agamben, G. (2010). Homo Sacer. El poder soberano y la nuda vida. Valencia, España: Pre-textos.

Allouch, J. (1998). El psicoanálisis, una erotología de pasaje. Córdoba: Litoral.

Aranguren. (1998), Ética. Barcelona: Altaya.

Benveniste, E. (1971). Problemas de lingüística general (vol. I-II). Madrid: Siglo XXI Editores.

Finley, M. I. (1961). El mundo de Odiseo. México: Fondo de Cultura Económica.

Foucault, M. (1999). Estética, ética y hermenéutica. Barcelona. Paidós.

Foucault, M. (2002). La hermenéutica del sujeto. México: Fondo de Cultura Económica.

Foucault, M. (2009/2010). El coraje de la verdad. Buenos Aires: Argentina: Fondo de Cultura Económica.

Foucault, M. (2011). Historia de la sexualidad (vol II). México: Siglo Veintiuno Editores. 
Hadot, P. (1998). ¿Qué es la filosofía antigua? México: Fondo de Cultura Económica.

Heidegger, M. (2000/2006). Carta sobre el humanismo (H. Cortés y A. Leyte, trads.). Madrid: Alianza Editorial. Recuperado de https:// escuelafilosofiaucsar.files.wordpress. com/2015/09/carta-sobre-el-humanismomartc3ad-heidegger.pdf

Homero. (2000). Odisea. Madrid: Gredos.

Huxley, A. (2014). Un mundo feliz. México: Ediciones del Sindicato Nacional de Trabajadores del Infonavit.

Lacan, J. (2009). El seminario XVII. La ética del psicoanálisis. Argentina: Paidós.

MacIntyre, A. (1991). Historia de la ética. Barcelona, España: Paidos.
Otto, W. F. (2003). Los dioses de Grecia. Madrid: Siruela.

Rajchman, J. (2001). Lacan, Foucault y la cuestión de la ética. México: Editorial Psicoanalítica de la letra.

Rojas, J. (2014). Sujeto del eros, sujeto del êthos: la genealogía del sujeto del deseo. [Con] textos, 3(11), 11-18.

Rojas, J. (2015). El gesto cínico. Notas sobre la política que viene. Revista Cientifica Guillermo de Okham, 13(2), 129-125. https://doi. org/10.21500/22563202.2071

Sampson, A. (1998). Ética, moral y psicoanálisis. Revista Colombiana de Psicología, (7), 81-93.

Vega, J. (2000). La astucia de la razón en la técnica. Arbor, 167(57), 187-205. 